It is less easy to account for the appearance of many monotypic endemic genera.

Dr. E. Fischer-Piette (Paris) gave an account of his work on the limpets of the North Atlantic. These form a complex capable of splitting into several species. This splitting is realized, morphologically and ecologically, in certain districts of the coastline, but not in others. In these last districts there is much variation, leading to unaccustomed types each special to a locality. Geographical isolation is not necessary for the formation of new species of limpets, although their formation is greatly favoured by it.

Dr. Bernard Rensch (Münster) opposed the view put forward by Goldschmidt, among others, that the transformation of species is governed by special laws, whereas geographical races only result from a secondary splitting up of species. He considers that geographical isolation of large populations is the first step not only towards new races but also towards new species, and that such a differentiation gives rise also to the higher systematic categories. The separation of new species by isolation from race-complexes (Rassenkreise) is the chief method of evolution. Examples of other methods are not numerous. Sometimes ecological isolation has been at work and, more rarely, a dominant mutation arises and spreads until it replaces the original form. In other cases, selection by external factors, especially climatic ones, is evident, leading to parallelism among geographical races. Prof. Karl Skottsberg discussed the flora of the Juan Fernandez group of islands and of the Hawaiian group.

In both symposia, more especially the first, the various speakers approached the problem of the nature and origin of species from very diverse points of view-systematic, palæontological, ecological, genetic, cytological. There resulted a certain appearance of discontinuity in the discussion, which, as one of the speakers remarked, emphasized the desirability of combined and detailed explora. tion of the field towards which all these lines of research converge. For such exploration, no more fitting place could be found than the Linnean Society.

\title{
Molecular Migration under the Influence of Centrifugal, Osmotic and Electric Forces*
}

$\mathrm{O}^{\mathrm{u}}$ UR knowledge of the mass movements of large molecules in various fields of force has its foundation in the early researches of Svedberg, and consists in large part in contributions from the Uppsala School. Since the occasion of Svedberg's recent lectures at Oxford $\dagger$, further improvements in experimental technique have been made, and the scope of these physical investigations, particularly where they touch on biological problems, has been greatly enlarged.

Studies of the resolving power of centrifuge rotors reveal that, in general, rotors of large diameter rotating at low speeds are preferable to smaller rotors at higher speeds. The resolving power is slightly higher and greater accuracy of optical observation is possible with the larger rotors. For rotor construction, steel is still to be preferred to light metal alloys, in spite of the greater ratio of strength to density offered by the newer aircraft alloys. This is due to the fact that around the cell holes, about one half of the total stress arises from the outward thrust of the cell, and the cell weight in modern constructions is

- An account of three lectures delivered by Prof. The Svedberg, of Uppsala, at University College, London, on April 29 , May 2 and 3 . + Summarized in a supplement to NATURE of June 19, 1937 reduced to a minimum. By reducing the bearing diameter from $18 \mathrm{~mm}$. to $10 \mathrm{~mm}$., the power dissipated in bearing friction has been reduced to some 300 watts at 60,000 revolutions per minute, or about one seventh of the earlier value. Rotors with such low bearing friction take an inconveniently long time to come to rest, and to reduce this time a magnetic brake system has been introduced. In spite of the much lower frictional losses in the air turbine ultracentrifuge, the energy required to drive this type is now actually greater than in the case of the oil turbine machine, owing mainly to the much lower efficiency of the air turbines. A new type of divided centrifuge cell, the inner chamber of which is made from the British transparent plastic 'Perspex', is available which enables the observation and separation in substance of ultracentrifuge fractions at the end of an experiment.

The study of molecular migrations in the electric field has been facilitated by the development of an improved electrophoresis apparatus by Tiselius in Svedberg's laboratory. It is possible with this apparatus to observe the migration of the com. ponents of a mixture and to isolate these for subsequent chemical or biological investigation. 
By application of the methods of sedimentation, osmotic and electrophoretic analysis to many naturally occurring substances, much valuable information has been obtained, and certain general conclusions may be drawn regarding the molecular state of substances in Nature. Of the proteins it may be said that Nature prefers to manufacture these in a homogeneous form, that is, with molecules all of the same size. Good examples of.homogeneous proteins are the albumin of the hen's egg, the crystalline globulin of milk, and the coloured respiratory proteins. A protein is only homogeneous within certain limits of hydrogen ion concentration of its solution. Outside these limits, associations and dissociations of the molecules take place. In the case of the hæmocyanin pigment from the snail Helix pomatia, for example, dissociation of the large parent molecule occurs into fragments of one half, one eighth and one sixteenth of the molecular weight of the original. Numerous other factors cause similar step-wise degradation of protein molecules, the changes being often of an irreversible nature. Of special interest is a form of specific degradation which is, however, reversible. It appears that in a mixture of proteins the larger molecules are frequently split up by the influence of the smaller molecules. Blood serum affords an important illustration of this, the concentration of smaller molecules in the undiluted serum being increased markedly by dissociation of the larger globulin molecules into fragments of the approximate weight of serum albumin, namely, 69,000. It is not possible to give a complete explanation of this phenomenon, which must be of great importance in the living organism.

Proteins which are homogeneous in the centrifuge are frequently also homogeneous in the electrophoresis apparatus. A notable exception is the globulin of blood serum, the molecules of which are approximately uniform in size with a weight in the neighbourhood of 160,000 . In the electric field these molecules separate into three distinct groups of different mobilities. Tiselius has been able to isolate these globulins and to characterize them as protein entities.

Various diseases alter the state of the serum proteins, and it is well known that in the majority of cases the alteration affects only the globulin part of the serum. These new methods show that in the serum obtained by immunizing the rabbit with egg albumin, only one of the three normal globulins is increased. Separation of this globulin results in a considerable concentration of specifically precipitable protein. Immunization of the rabbit with type III pneumococcus results in the appearance of a new serum protein with the same molecular weight as the normal rabbit globulins but electrochemically different from these. On absorp. tion of the serum with the type specific carbohydrate, this new fraction can be removed quantitatively from the electrophoresis photograph. On the other hand, immunization of the horse with type I pneumococcus produces a fraction which is different both in respect of molecular weight and electrophoretic mobility from the normal globulins. These and other phenomena demonstrate that the changes in the serum proteins which accompany the development of immunity are of a diverse nature and are not at the moment amenable to any simple explanation.

In the important field of the respiratory proteins, cytochrome $C$ and myoglobin have been found to have the same molecular weight, namely 17,000 , or approximately one fourth of the weight of hæmoglobin. The yellow enzyme, on the other hand, has a molecular weight of 80,000 and one prosthetic group in the molecule. All the enzymes so far isolated in a pure state have been proved to be proteins. Pepsin is a well-defined protein of molecular weight 37,000 . The catalase molecule has recently been shown to contain four atoms of iron in the molecule, like hæmoglobin, but to have a molecular weight approximately four times as great, namely, 248,000 . The largest enzyme molecule so far measured is that of crystalline urease, which has a weight of 483,000 .

In recent years, more attention has been devoted to systems containing linear macromolecules. This field includes the carbohydrates, particularly cellulose, starch and glycogen ; synthetic polymers such as the polystyrenes; and hydrocarbons such as rubber. In general these systems are far from homogeneous, the chain length varying from one molecule to another. The main object of study, therefore, is the size-distribution curve, which is best obtained from measurements of sedimentation velocity. Suitable solvents are often difficult to find, and the interpretation of the results is complicated by the asymmetry of the molecules, by the interactions of neighbouring molecules, and by solvation. The molecular state of starch is much influenced by the past history of the preparation. Acid-treated solutions show an amylose fraction of mean molecular weight about 60,000 and an amylo pectin of approximately 200,000 ; both are polydisperse. Glycogen and its methylated derivatives are highly polymerized, and also polydisperse. The specific polysaccharide of type I pneumococcus is polydisperse with an average weight of approximately 225,000 . Recently, however, a homogeneous polysaccharide of low molecular weight, namely, 9,000 , has been isolated by Dr. Florence Seibert from filtrates of tubercle bacillus cultures.
A. S. McFarlane. 